\title{
RESEARCH
}

\section{Steroid receptor coactivator-3 as a target for anaplastic thyroid cancer}

\author{
Woo Kyung Lee1, Won Gu Kim¹,2, Laura Fozzatti13,3, Sunmi Park¹, Li Zhao1', Mark C Willingham¹, David Lonard4, \\ Bert W O'Malley4 and Sheue-yann Cheng 1
}

1Laboratory of Molecular Biology, Center for Cancer Research, National Cancer Institute, National Institutes of Health, Bethesda, Maryland, USA 2Division of Endocrinology and Metabolism, Department of Internal Medicine, Asan Medical Center, University of Ulsan College of Medicine, Seoul, Korea ${ }^{3}$ Departamento de Bioquímica Clínica, Facultad de Ciencias Químicas, Universidad Nacional de Córdoba, Córdoba, Argentina

${ }^{4}$ Department of Molecular and Cellular Biology, Baylor College of Medicine, Houston, Texas, USA

Correspondence should be addressed to S Cheng: chengs@mail.nih.gov

\begin{abstract}
Anaplastic thyroid carcinoma (ATC) is an aggressive malignancy without effective therapeutic options to improve survival. Steroid receptor coactivator-3 (SRC-3) is a transcriptional coactivator whose amplification and/or overexpression has been identified in many cancers. In this study, we explored the expression of SRC-3 in ATCs and the effects of a new class of SRC-3 inhibitor-2 (SI-2) in human ATC cells (THJ-11T and THJ-16T cells) and mouse xenograft models to assess therapeutic potential of SI-2 for the treatment of ATC. SRC-3 protein abundance was significantly higher in human ATC tissue samples and ATC cells than in differentiated thyroid carcinomas or normal controls. SI-2 treatment effectively reduced the SRC-3 expression in both ATC cells and ATC xenograft tumors induced by these cells. Cancer cell survival in ATC cells and tumor growth in xenograft tumors were significantly reduced by SI-2 treatment through induction of cancer cell apoptosis and cell cycle arrest. SI-2 also reduced cancer stem-like cells as shown by an inhibition of tumorsphere formation, ALDH activity, and expression of stem cell markers in ATC. These findings indicate that SRC-3 is a potential therapeutic target for treatment of ATC patients and that SI-2 is a potent and promising candidate for a new therapeutic agent.
\end{abstract}

\section{Key Words}

- anaplastic thyroid carcinoma

- steroid receptor coactivator-3

- small-molecule inhibitor

- cancer stem-like cells

- $\mathrm{SI}-2$

\section{Introduction}

Anaplastic thyroid carcinoma (ATC) is a rare malignancy, accounting for $1-2 \%$ of all thyroid cancers, but it is one of the most aggressive solid cancers in humans (Molinaro et al. 2017). ATC is highly proliferative, easily invades the trachea, and metastasizes to distant organs. The median survival of patients with ATC is only 3-5 months after diagnosis (Smallridge 2012, Jeon et al. 2016, Tiedje et al. 2018). Unlike the differentiated thyroid carcinoma (DTC) such as papillary thyroid carcinoma (PTC) and follicular thyroid carcinoma (FTC), ATC cells do not have any functional and biological features of normal thyrocytes, such as thyrotropin dependence, iodine uptake, and thyroglobulin synthesis (Molinaro et al. 2017). ATC is usually resistant to radioactive iodine therapy or chemotherapy and there are no established therapeutic options to improve overall survival of patients (Smallridge 2012, Tiedje et al. 2018). American Thyroid Association (ATA) guidelines and the National Comprehensive Cancer Network and Practical Guidelines for ATC suggest combination therapy including surgery, radio-, and/or
(C) 2020 Society for Endocrinology Published by Bioscientifica Ltd. Printed in Great Britain 
chemotherapy to maximize clinical benefits (Smallridge et al. 2012, Haddad et al. 2015). Currently, several new tyrosine kinase inhibitors (TKIs) have been tested or are under evaluation in phase II clinical trials. Only a combination of dabrafenib and trametinib is approved by US Food and Drug Administration for patients with $B R A F$ mutated ATC based on the results of a phase II clinical trial without definite evidence of survival benefits (Subbiah et al. 2018). A novel therapeutic modality for the treatment is therefore urgently needed.

Steroid receptor coactivator-3 (SRC-3, official gene name, NCOA3; also known as AIB1, RAC3, pCIP, ACTR, and TRAM1) is an important transcriptional coactivator which modulates transcriptional activity of the nuclear receptor superfamily and other transcription factors (Wang et al. 2016). SRC-3 was initially identified as a transcriptional coactivator amplified and overexpressed in estrogen receptor-positive breast and ovarian cancer (Anzick et al. 1997). Subsequently, SRC-3 amplification and/or overexpression was found in various types of hormonedependent and hormone-independent cancers such as breast cancer, endometrial carcinoma, ovarian cancer, prostate cancer, colorectal cancer, esophageal squamous cell carcinoma, gastric cancer, hepatocellular carcinoma, meningioma, and pancreatic cancer (Yan et al. 2006, Xu et al. 2009, Wang et al. 2016). Mice over-expressing SRC-3 developed malignant mammary, pituitary, and uterine tumors by activating insulin growth factor 1 (IGF-1) and phosphinositide 3 kinase (PI3K)/AKT signaling pathway (Torres-Arzayus et al. 2004). Previously, we have created a mutant mouse that expresses dominate-negative mutant thyroid hormone receptor $\beta$ mutant (TR $\beta \mathrm{PV}$ ). This mutant mouse (ThrbPV/PV mice) spontaneously develops follicular thyroid cancer with pathological progression and frequency of metastasis is similar to that in cancer patients (Suzuki et al. 2002). Much like tumors in patients, the development of thyroid tumors in $T h r b^{P V / P V}$ mice is propelled by over-activation of PI3K-AKT signaling (Kim et al. 2005, Guigon et al. 2009). Interestingly, Thrb $b^{P V / P V}$

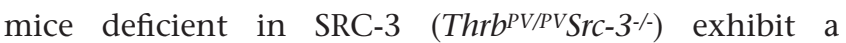
significant increased survival, decreased thyroid tumor growth, delayed tumor progression, and markedly lower incidence of distant metastasis as compared with $T h r b^{P V / P V}$ mice with SRC-3 (Thrb ${ }^{\text {PV/PV SrC-3 }}{ }^{+/+}$) (Ying et al. 2008). These findings clearly indicate that SRC-3 is a tumor promoter in thyroid cancer and, importantly, that SRC-3 could be a potential therapeutic target of thyroid cancer.

Many small molecule inhibitors (SMIs) have been identified and developed for targeting mainly the enzyme substrate binding site of kinases (Wells \& McClendon 2007).
However, transcriptional co-activators are considered to be more difficult molecules to target because of their large size and disordered structures (Lonard \& O'Malley 2006, 2012). Recently, Song et al. reported the development of a promising new class of SRC-3 inhibitor-2 (SI-2) by using cell-based functional assays for high-throughput screening (Song et al. 2016). SI-2 can selectively inhibit transcriptional activities of SRC-3 and the protein concentrations of SRC-3 in breast cancer cells in vitro and breast cancer tumors in xenograft models (Song et al. 2016).

In the present study, we showed that SRC-3 expression was significantly increased in human ATC tissues samples and ATC cell lines. SI-2 treatment reduced protein abundance of SRC-3 in ATC cells and xenograft tumors. Cancer cell survival of ATC cells and tumor growth of xenograft models were inhibited by SI- 2 treatment through induction of apoptosis and suppression of cell-cycle progression. We also found that SI-2 treatment inhibited the cancer stem-like cells (CSCs) in ATC. These findings indicate that SRC-3 is a potential therapeutic target for ATC and that SI- 2 is a potent and promising candidate as a new therapeutic agent for patients with ATC.

\section{Materials and methods}

\section{Cell lines}

All THJ cell lines derived from ATC (THJ-11T, THJ-16T, THJ-21T, and THJ-29T) were provided by Dr John A Copland III at the Mayo Foundation for Medical Education and Research (Marlow et al. 2010). These cells were maintained in RPMI-1640 media (Invitrogen) supplemented with 10\% fetal bovine serum (FBS), 1\% non-essential amino acid, 1\% sodium pyruvate, and $1 \%$ antibiotic-antimycotic solution (Thermo Fisher Scientific), as previously described (Zhu et al. 2019). FTC-133 and FTC-236 cells derived from follicular thyroid carcinoma (FTC), kindly provided by Dr Orlo H Clark (UCSF Mount Zion Medical Center, San Francisco, CA), were maintained in DMEM/Ham's F12 (1:1) medium (Invitrogen) supplemented with 10\% FBS, $10 \mu \mathrm{g} / \mathrm{mL}$ bovine insulin (Sigma-Aldrich), $1 \mathrm{mIU} / \mathrm{mL}$ bovine TSH (Sigma-Aldrich), $100 \mathrm{U} / \mathrm{mL}$ penicillin, and $100 \mu \mathrm{g} / \mathrm{mL}$ streptomycin (Invitrogen), as previously reported (Kim et al. 2013).

\section{Cell survival assays}

SI-2 was provided by Dr Bert W O'Malley, Baylor College of Medicine, Houston, Texas (Song et al. 2016).
(C) 2020 Society for Endocrinology Published by Bioscientifica Ltd. Printed in Great Britain 
To evaluate the effects of SI- 2 on cell proliferation, cells were cultured in the media with various concentrations of SI-2 or DMSO in six-well plates each with triplicates, as previously described (Kim et al. 2014). The number of viable cells was counted at $48 \mathrm{~h}$ after treatment using a cell counter (Countess II, Thermo Fisher Scientific). The relative cell viability was calculated after normalization of DMSO-only control.

\section{Western blot analysis}

Preparation of whole-cell lysates and Western-blot analysis were carried out as previously described (Furumoto et al. 2005). The protein samples were loaded and separated by sodium dodecyl sulfate PAGE. After electrophoresis, the protein was electrotransferred to a polyvinylidene difluoride membrane (Immobilon-P; Millipore). Antibodies against SRC-3 (\#2126, Cell Signaling, Inc.), P21 (\#2947, Cell signaling), Cyclin D1 (RB-9041-P1, Thermo Scientific), E2F1 (sc-251, Santa Cruz), Bad (\#9292, Cell signaling), Bim (\#2933, Cell signaling), and cleaved poly ADP ribose polymerase (PARP) (\#5625, Cell signaling) were used. The blots were stripped with Re-Blot Plus (Chemicon) and re-probed with antibody against $\beta$-actin (\#4970, Cell signaling). Band intensities were quantified by using NIH Image software (ImageJ; Wayne Rasband, NIH, Bethesda, MD).

\section{Flow cytometry analysis}

For each analysis, THJ-11T and THJ-16T cells were first incubated with the indicated dose of SI-2 or vehicle for 24 or $48 \mathrm{~h}$. For cell cycle analysis, the cells were trypsinized, washed, and fixed in $70 \%$ ethanol at $-20^{\circ} \mathrm{C}$ overnight. On the next day, the cells were washed and then incubated in DAPI staining solution (D9542, Sigma-Aldrich: $1 \mu \mathrm{g} / \mathrm{mL}$ DAPI in PBS) for $10 \mathrm{~min}$ at room temperature. Cell apoptosis was measured with Annexin V-FITC apoptosis detection kit (556547, BD Biosciences). To assay for ALDH activity, we used an ALDEFLUOR kit (\#01700, Stem Cell Technology, Ltd.) as per the manufacturer's protocol. Briefly, the cells were trypsinized, after which 1 million cells were resuspended in $1 \mathrm{~mL}$ aldeflour assay buffer. Aldeflour reagent was added to this cell suspension and then half of the cell suspension was transferred to a fresh tube containing diethylaminobenzaldehyde (DEAB), which is used to control background fluorescence as a specific inhibitor of ALDH. The cells were incubated at $37^{\circ} \mathrm{C}$ for $30 \mathrm{~min}$, washed, and then subjected to flow cytometry. All the stained cells were analyzed by Sony

(c) 2020 Society for Endocrinology Published by Bioscientifica Ltd. Printed in Great Britain
SA3800 spectral cell analyzer (Sony Biotechnology, Inc.). The data analysis was done by FlowJo software 10.5.3 (FlowJo LLC).

\section{Analysis of TCGA data}

A public transcriptome database matched to clinicopathological data from The Cancer Genome Atlas (TCGA-THCA, http://cancergenome.nih.gov/: thyroid cancer $(n=505)$ and normal thyroid tissue $(n=59))$ was used to identify biological characteristics and clinical significance of thyroid cancer with high expression of the NCOA3 gene encoding SRC-3 protein.

To identify the role of NCOA3 gene for cancer stemness, we used 'Stemness Index', generated by a machine-learning algorithm based on both transcriptomic and epigenetic signatures of stem cells (embryonic stem cells and induced pluripotent stem cells) to analyze TCGA dataset as previously reported (Malta et al. 2018). For gene set enrichment analysis (GSEA, http://software. broadinstitute.org/gsea/index.jsp) and clinicopathological data analysis, we used the transcriptome data of tumors with the top 30 and the bottom 30 NCOA3 expression in the TCGA-THCA: low and high NCOA3 group $(n=30$, respectively). GSEA predicted coordinated upregulation or downregulation of pre-organized signature gene sets, such as the Kegg and Hallmark gene sets, in the high NCOA3 group vs the low NCOA3 group. To examine the relationship of NCOA3 with cancer driver- and pathwayrelated genes, we created our own gene set including 48 cancer-related genes, as previously reported (Mlecnik et al. 2016). In our setting, a negative normalized enrichment score (NES) indicates gene set enrichment at the top of the ranked list in the high NCOA3 group. Among the enriched gene sets in the group, those with a nominal $P$-value $<0.05$ and an FDR $q$-value $<0.25$ were considered statistically significant.

\section{In vivo mouse xenograft study}

The National Cancer Institute Animal Care and Use Committee approved the protocols for animal care and handling in this study. Four-week-old female athymic NCr-nu/nu mice were obtained from the NCI-Frederick animal facility. THJ-11T and THJ-16T cells $\left(5 \times 10^{6}\right.$ cells) in $200 \mu \mathrm{L}$ of suspension mixture with MatrigelBasement Membrane Matrix (354234, Corning) were subcutaneously inoculated into the right flank of mice, as previous reported (Kim et al. 2013). After 3 weeks, mice were randomly divided into the treatment and control 
groups. The treatment group received an intraperitoneal injection of SI-2, $2 \mathrm{mg} / \mathrm{kg}$ per dose, two doses per day, for 2-6 weeks, while the control group received vehicle solution, as previously reported (Song et al. 2016). Tumor size was measured with calipers, and tumor volume (V) was calculated as (length $\times$ width $\left.^{2}\right) / 2$. Each experiment ended when the length of the biggest tumor reached 20 $\mathrm{mm}$. Tumor weight was determined after dissection of xenografted tumor tissues from the killed mice at the study endpoint.

\section{Immunohistochemistry and histopathological analysis}

Formalin-fixed, paraffin-embedded (FFPE) tissues from surgically removed human thyroid cancer samples were collected at Asan Medical Center (Seoul, Korea), between 1996 and 2012 following the Institutional Review Board approval. The use of human samples was also approved by the IRB board of the National Cancer Institute. Tissues from xenografted tumor were fixed in 10\% neutral buffered formalin (Sigma-Aldrich) and subsequently embedded in paraffin. Five-micrometer-thick sections were prepared and stained with hematoxylin and eosin. Immunohistochemistry (IHC) was performed as previously described (Kim et al. 2012) with some modification using Vectastain ABC kit (Vector Laboratories). Antibodies for SRC-3 (\#611104, BD Transduction Laboratories), Cyclin D1 (RB-9041-P1, Thermo Scientific), Ki-67 (RB-9043-P0, Thermo Scientific), Bim (\#2933, Cell Signaling), cleaved caspase-3 (\#9664, Cell Signaling), ALDH1/2 (sc-166362, Santa Cruz), and $\beta$-catenin (\#9562, Cell Signaling) were used to evaluate protein expression by IHC.

\section{Tumorsphere assay}

Cells were plated in 96-well low attachment plates (Corning) in DMEM/F12 (1:1) with 1\% methylcellulose (Sigma), bFGF (Peprotech), hEGF (R\&D Systems), B27 supplement (Life Technologies), and penicillin/ streptomycin (Gibco). Fresh media was added every 3 days, and the number of tumorspheres were counted and photographed after 10 days. A Zeiss light microscope was used to count and quantify the tumorspheres (Rohira et al. 2017).

\section{Statistical analysis}

Data are presented as mean \pm s.D. Student $t$-test or oneway analysis of variation with Tukey's post-hoc test was used to compare continuous variables between groups. Categorical data were compared using the chi-squared test or Fisher's exact test. Two-way ANOVA with Bonferroni's post-hoc test was used to compare tumor growth in xenograft model according to the time and groups. To identify the relationship of $N C O A 3$ with cancer stemnessrelated genes in the TCGA-THCA, we used Pearson correlation coefficient for the analyses. For schematization of the results from these analyses, we used RStudio 1.2 (RStudio Inc., Boston, MA, USA). P-values were two-sided throughout and less than 0.05 was considered statistically significant. Data were analyzed using GraphPad Prism 8.1.1 (GraphPad Software).

\section{Results}

\section{SI-2 inhibits ATC cell survival by inducing apoptosis and by impeding cell cycle progression}

To elucidate the effects of SI-2 on ATC cells, we first evaluated the protein abundance of SRC-3 in four human cell lines derived from ATC tumors of patients (THJ-11T, THJ-16T, THJ-21T, and THJ-29T) (Marlow et al. 2010), two FTC cell-lines (FTC-133 and FTC-236, derived from primary lesions and lymph node metastasis, respectively) (Hoelting et al. 1994), and normal controls (101N and $116 \mathrm{~N})$. SRC-3 protein abundance was increased in four THJ cells and FTC-236 as compared with normal controls (Fig. 1A-I). The relative protein abundance of SRC-3 was highest in THJ-11T (2.8-fold increase) and THJ-16T (2.5-fold increase) compared with normal control, $116 \mathrm{~N}$ (Fig. 1A-II). Therefore, we used the two human ATC cells (THJ-11T and THJ-16T) for further experiments.

Previous studies showed that SI-2 inhibited SRC-3 protein abundance (Song et al. 2016). Therefore, we first investigated the effects of SI-2 on SRC-3 protein abundance in ATC cells. Cellular protein levels of SRC-3 were significantly decreased in a dose-dependent manner after SI-2 treatment in both THJ-11T and THJ-16T cells (Fig. 1B-I and quantitation in Fig. 1B-II). Concurrently, SI-2 treatment reduced the number of viable cells in a dose-dependent manner in THJ-11T and THJ-16T cells (Fig. 1C-i and -ii, respectively). We performed an apoptosis assay using Annexin $\mathrm{V}$ and propidium iodide to examine the cause of the SI-2 cytotoxic effect on these ATC cells. The percentage of cells in early and late apoptotic phases was increased after SI-2 treatment (Fig. 1D-I). The quantitative analysis revealed that the relative fold of 


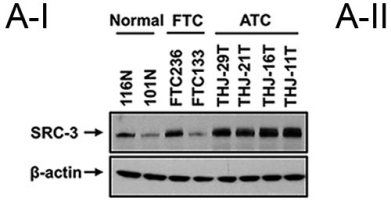

C
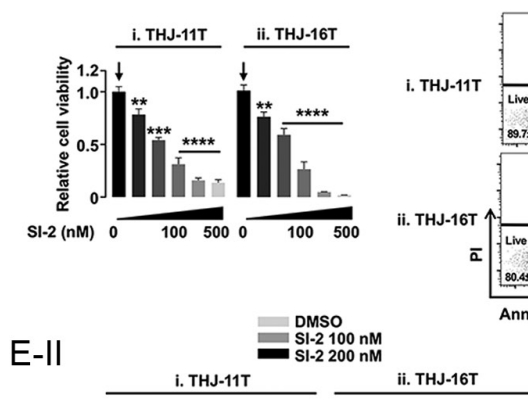

D-I
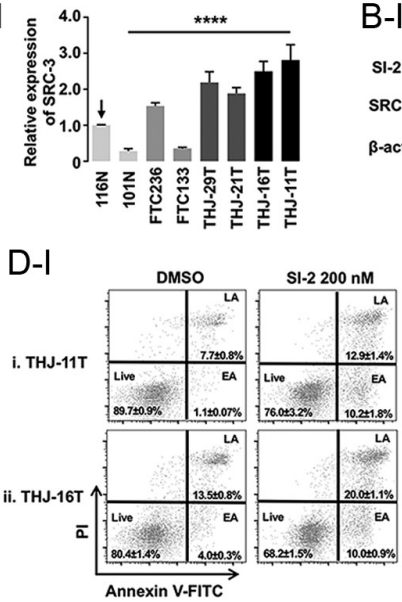

F-I
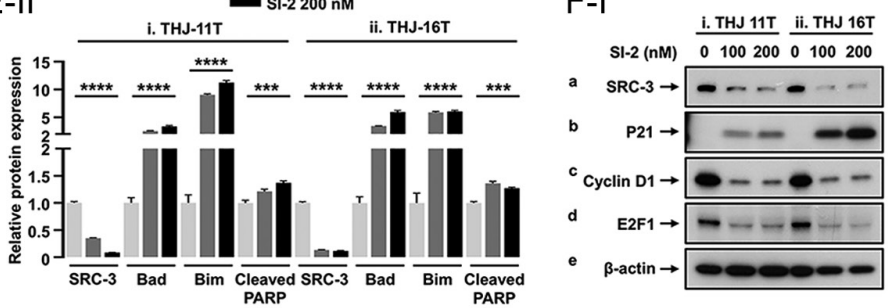

B-I

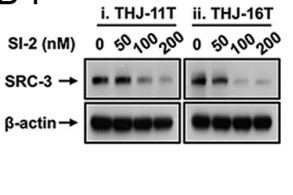

D-II

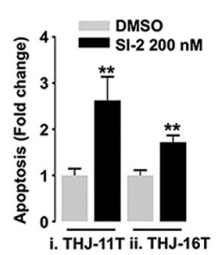

B-II

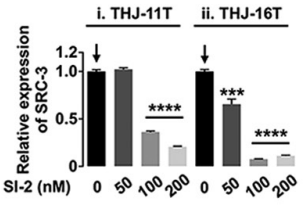

E-I

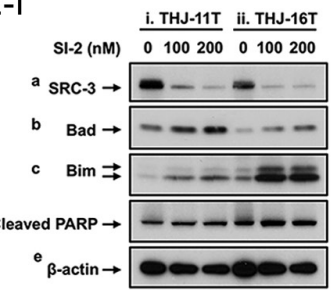

F-II

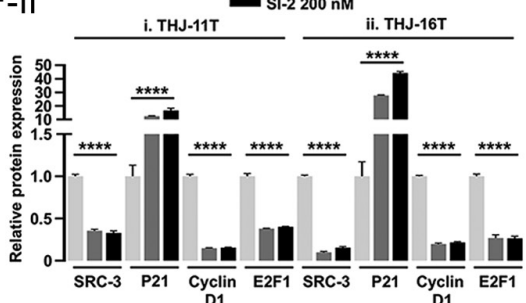

\section{Figure 1}

SI-2 suppresses proliferation through induction of apoptosis and cell cycle arrest in ATC cells. (A) Western blot (I) and its quantitative analysis (II, $n=3$ ) showing different expression levels of SRC-3 protein in normal thyroid, FTC, and ATC cell lines. (B and C) Western blot (B-I) and its quantitative analysis (B-II, $n=3$ ) showing dose-dependent inhibition of SRC-3 protein (B), and dose-dependent inhibition of cell proliferation $(C, n=3)$ by 48 -h treatment of SI-2 in ATC cells. (D) Annexin V apoptosis assay (I) and its quantitative analysis (II, $n=3$ ) showing effect of SI-2 treatment on apoptosis in ATC cells. (E) Western blot (I) and its quantitative analysis (II, $n=3)$ showing effect of SI-2 treatment on protein expression of apoptosis-related molecules in ATC cells. (F) Western blot (I) and its quantitative analysis $(I I, n=3)$ showing effect of SI-2 on protein expression of cell cycle-related molecules in ATC cells. Significant differences are indicated by asterisks $(P<0.01(* *), P<0.001(* * *)$, and $P<0.0001(* \star \star *))$. Data represent the mean \pm S.D. FTC, follicular thyroid cancer; ATC, anaplastic thyroid cancer.

increase in apoptosis was 2.6-fold for THJ-11T cells and 1.7-fold for THJ-16T cells (Fig. 1D-II-i and -ii). We further identified the molecular changes that led to the increase of apoptosis caused by SI-2 treatment.

As shown in the Western blot analysis, concurrent with the dose-dependent decrease in SRC-3 protein levels (Fig. 1E-I-a), the protein levels of key apoptotic regulators, Bad (panel b), Bim (panel c), and marker of apoptosis, cleaved PARP (panel d), were induced accordingly in both THJ-11T and THI-16T cells (also see the quantitation in Fig. 1E-II). These data indicated that SI-2 treatment inhibited ATC cell survival by inducing apoptosis.

Decreased cell survival also could be due to inhibition of cell proliferation by delaying cell cycle progression. We therefore used Western blot analysis to analyze several critical cell cycle regulators in THJ-11T and THJ-16T after treatment with SI-2. The cyclin-dependent kinase inhibitor p21 (also known as p21 WAF1/Cip1) was increased (Fig. 1F-I-b) concurrently with the decreased SRC-3 (Fig. 1F-I-a) in the two ATC cell lines by treatment with SI-2 at $100-\mathrm{nM}$ or $200-\mathrm{nM}$ concentrations. The increase in p21 was accompanied by a concurrent decrease in cyclin D1 protein levels (Fig. 1F-I-c). The transcription factor E2F1 plays a crucial role in the control of the cell cycle by coordinating the gene expression involved in DNA replication and cell cycle progression (Nevins et al. 1997, Trimarchi \& Lees 2002). Previous studies indicated that SRC-3 directly interacts with E2F1 for upregulation of E2F1 target gene expression via a positive regulatory loop between E2F1 and SRC-3 (Louie et al. 2004, Mussi et al. 2006). The protein abundance of E2F1 by SI-2 treatment was dose-dependently decreased (Fig. 1F-I-d). Quantitative analysis indicated that SI-2 treatment significantly decreased protein abundance of cyclin D1 and E2F1, ranging from $60 \%$ to $85 \%$ in both ATC cell lines (Fig. 1F-II). These data indicate that SI-2 treatment in ATC cells impeded cell cycle progression. Taken together, these results show that SI-2 decreased ATC cell survival by both induction of apoptosis and impeding cell cycle progression. 


\section{SI-2 inhibits growth of tumors induced by} THJ-11T and -16 cells in mouse xenograft models

To further analyze the inhibitory effects of SI-2 on ATC tumor cell growth, we next assessed the SI-2 effects on ATC cell-induced tumors in in vivo mouse xenograft models.

The growth of tumors-derived from THJ-11T cells was significantly inhibited by SI- 2 treatment $(n=7)$ as compared with vehicle treatment $(n=8)$ (Fig. 2A-I, see also 2B-I). Similarly, the growth of tumors-derived from THJ-16T cells was inhibited by SI-2 treatment $(n=8)$ (Fig. 2A-II, see also 2B-II). The mean tumor weight was reduced by $76.0 \%$ and $70 \%$ in SI-2 treated group as compared with the vehicle-treated control group at the study endpoint for THJ-11T and THJ-16T cell-induced tumors, respectively (Fig. 2C-I and -II). However, we found no significant differences in body weights of the mice between SI-2treated and vehicle-treated group (Fig. 2D), suggesting that there were no potential overt side effects on mice. These findings clearly show the effectiveness of SI-2 on inhibiting tumor growth in vivo.

\section{SI-2 induces apoptosis and cell cycle arrest in ATC xenograft tumors}

To elucidate how SI-2 inhibited xenograft tumor growth, we analyzed the changes in protein abundance associated with apoptosis and cell cycle regulation. We first used immunohistochemical analysis to evaluate the inhibition of SRC-3 protein levels in THJ-11T and THJ-16T tumors after SI-2 treatment (Fig. 3A-I-i and -ii, respectively). There were significant decreases in the protein abundance of SRC-3 in SI-2 treated tumors consistent with the results of in vitro experiments using ATC culture cells. Quantitative analysis showed that the number of SRC-3-positively stained cells was 68\% and 95\% lower in THJ-11T and THJ-16T tumors treated with SI-2 than in vehicle-treated controls (Fig. 3A-II-i and -ii, respectively). The expression of pro-apoptotic protein, Bim, was significantly increased by SI-2 treatment in THJ-11T and THJ-16T tumors, consistent with the in vitro study (Fig. 3B-I-i and -ii, middle panel). Quantitative analysis showed that the number of Bim-positively stained cells was 10-fold and 9-fold higher in THJ-11T and THJ-16T tumors treated with SI-2 than in vehicle-treated controls (Fig. 3B-II-i and -ii, respectively). We also evaluated the SI-2 induced apoptosis by evaluating the expression of cleaved caspase- 3 in these tumors. Significant increases in the cleaved caspase- 3 proteins by SI-2 treatment (Fig. 3B-I, bottom panel, also see Fig. 3B-II, right panel) were detected. Inhibition of cell cycle progression by SI-2 treatment was also an important finding in THJ-11T and THJ-16T cells. The number of cells stained with cyclin D1 and nuclear proliferation marker Ki-67 was apparent (Fig. 3C-I, middle and lower panels, respectively). The quantitative data indicated that cyclin D1 and Ki-67 positively-stained cells were decreased after SI-2 treatment (Fig. 3C-II, left and right panels,
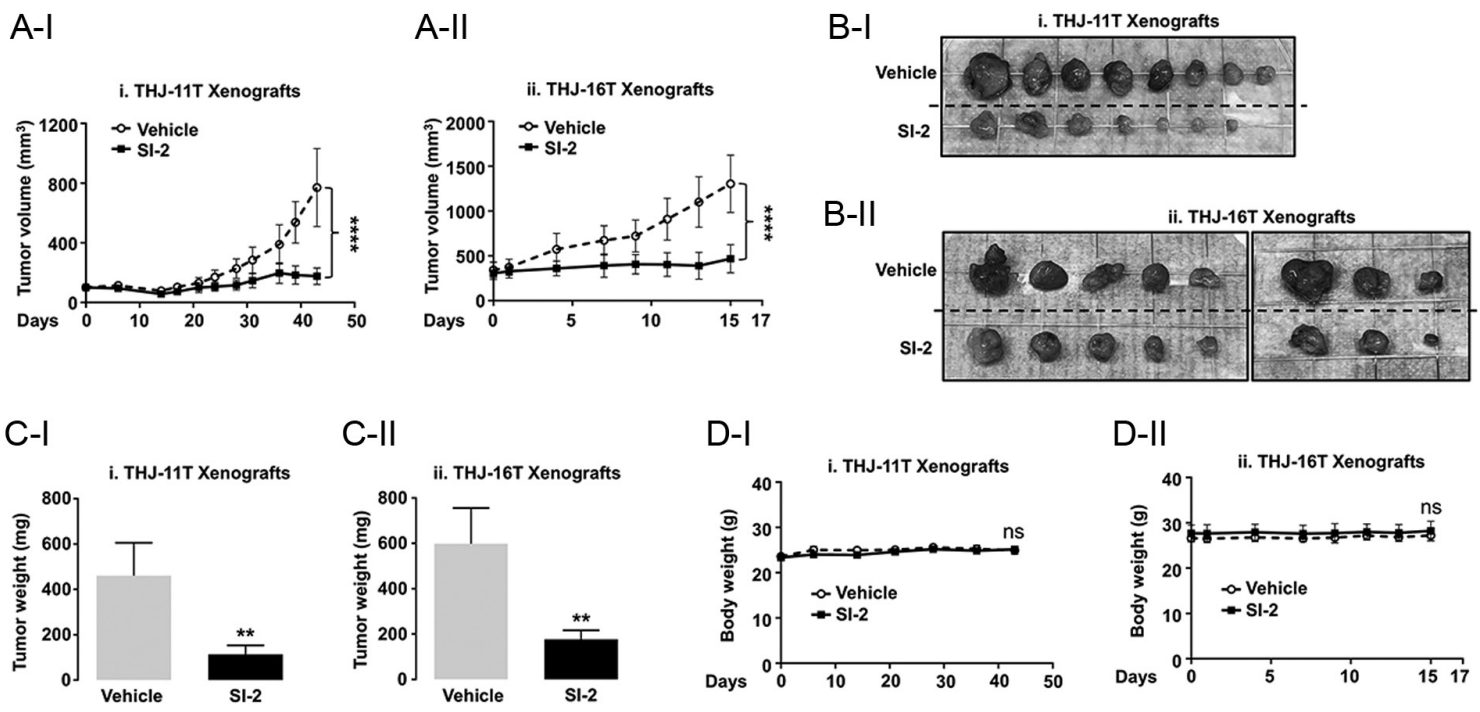

C-II

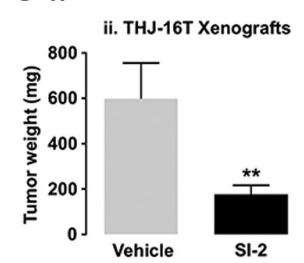

D-I
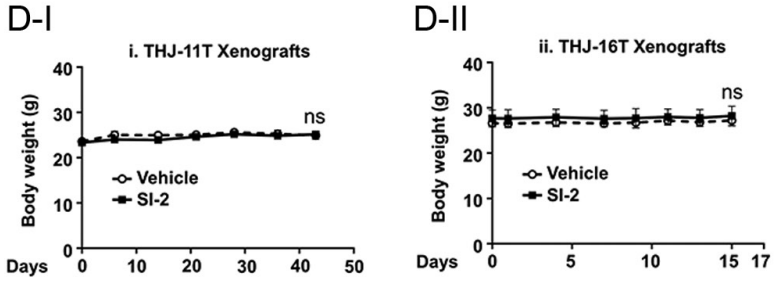

Figure 2

Therapeutic efficacy of SI-2 in ATC xenograft mice models. (A, B, and C) Growth curves (A), gross images (B), and weight (C) of THJ-11T (i) and THJ-16T (ii) xenograft tumors treated with vehicle or SI-2. (D) Change in the body weight of the mice. Significant differences are indicated by asterisks $\left(P<0.05\right.$ ( $\left.{ }^{\prime}\right)$ and $P<0.0001(* * * *))$. Data represent the mean \pm S.D. (For THJ-11T xenografts, vehicle group $(n=8)$ and SI-2 group $(n=7)$; For THJ-16T xenografts, both $\operatorname{groups}(n=8))$. 
A-I

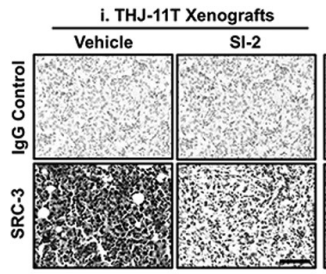

B-I

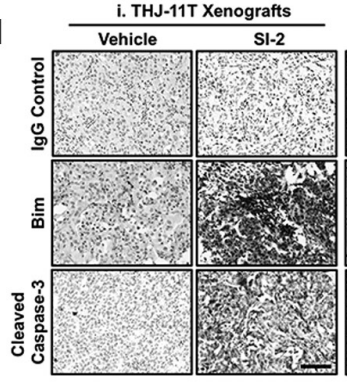

C-I

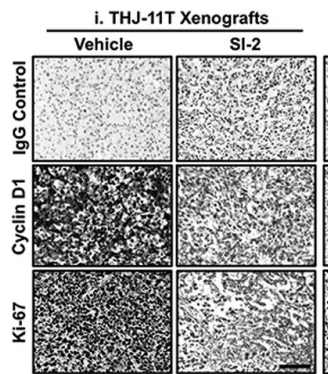

\begin{tabular}{|l|l} 
ii. THJ-16T Xenografts \\
\hline Vehicle
\end{tabular}

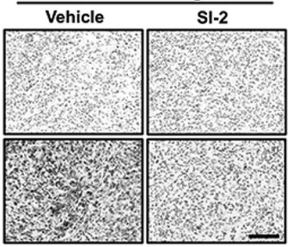

ii. THJ-16T Xenografts
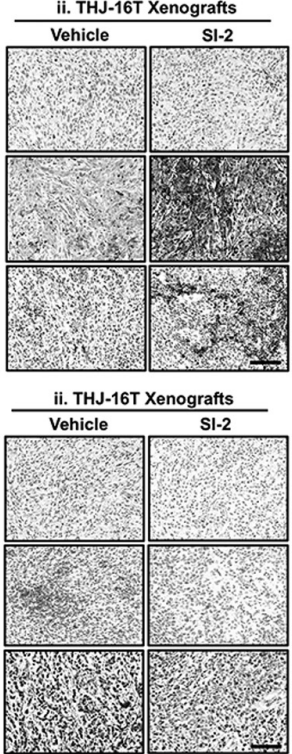

A-II

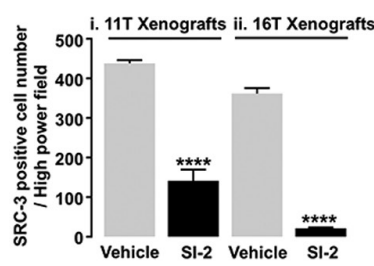

B-II
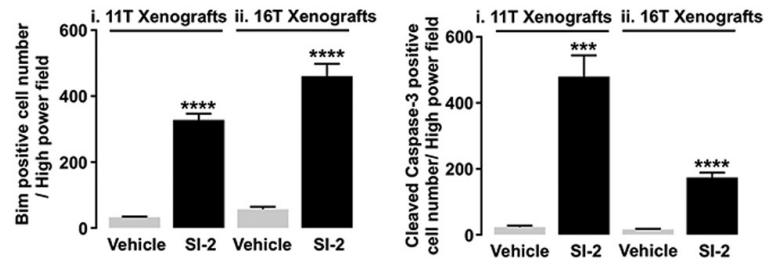

C-II
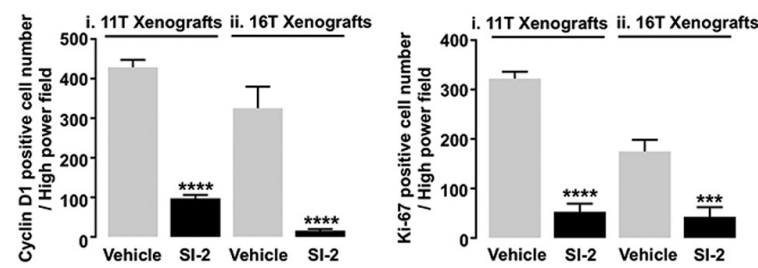

Figure 3

SI-2 effectively inhibits cell proliferation and induces apoptosis in vivo. (A) Representative IHC images for SRC-3 (I) and quantitative analyses for the IHC results (II, $n=4$, respectively) in the THJ-11T- and THJ-16T xenograft tumors treated with vehicle or SI-2. (B) Representative IHC images for molecules related to apoptosis (I) and quantitative analyses for the IHC results $(\mathrm{II}, n=4)$ in the THJ-11T and THJ-16T xenograft tumors treated with vehicle or SI-2. (C) Representative IHC images for molecules related to proliferation (I) and quantitative analyses for the IHC results (II, $n=4$, respectively) in the THJ-11T and THJ-16T xenograft tumors treated with vehicle or SI-2. Significant differences are indicated by asterisks $(P<0.001(* \star *)$ and $P<0.0001$ ( $(* \star *))$. Data represent the mean \pm s.D. Scale bar represents $50 \mu \mathrm{m}$. IHC, immunohistochemistry.

respectively). Consistent with in vitro findings, these data indicated that SI-2 treatment induced tumor cell apoptosis and cell cycle arrest in in vivo xenograft models of ATC.

\section{The effects of SI-2 on cancer stem-like cells in ATC}

Cancer stem-like cells (CSCs) are characterized by the ability of self-renewal, tumor initiation, and metastasis. They are a subpopulation of cells in tumors and play important roles in chemoresistance and recurrent cancers (Reya et al. 2001, Al-Hajj et al. 2003). A recent study suggested that SRC-3 is a regulator of stem-cell capacity in lung and breast cancer cells and is a potential therapeutic target for the prevention of cancer recurrence (Rohira et al. 2017). Accordingly, we next examined the effect of SI-2 on the cancer stemness of ATC cells. Interestingly, the TCGA data analysis revealed that the expression of NCOA3 gene encoding SRC-3 protein had strong positive correlation with genes related to cancer stemness in human thyroid cancer compared to normal thyroid tissue (Fig. 4A). The positive relationship of NCOA3 with cancer stemness was further analyzed by using 'Stemness Index', generated by a machine-learning algorithm based on both transcriptomic and epigenetic signatures of stem cells (embryonic stem cells and pluripotent stem cells) and applied to TCGA dataset as previously reported (Malta et al. 2018). The analysis showed that the Stemness Index was positively correlated with $\mathrm{NCOA} 3$ expression in TCGA-THCA data (Fig. 4B-I) significantly higher in tumor tissues with high NCOA3 expression than in tumors with low NCOA3 expression (Fig. 4B-II). The ALDEFLUOR assay clearly showed that SI-2 strongly suppressed intracellular ALDH activity in both THJ-11T and THJ-16T cells, indicating inhibition of CSCs function (Fig. 4C-I-i and -ii, respectively). Quantitative analysis showed that SI-2 treatment suppressed ALDH activity by $75 \%$ and $54 \%$ in THJ-11T and THJ-16T, respectively, as compared with controls (Fig. 4C-II). Consistently, in vitro tumorsphere formation assay showed that SI-2 dose-dependently suppressed tumorsphere formation of THJ-11T and 
A

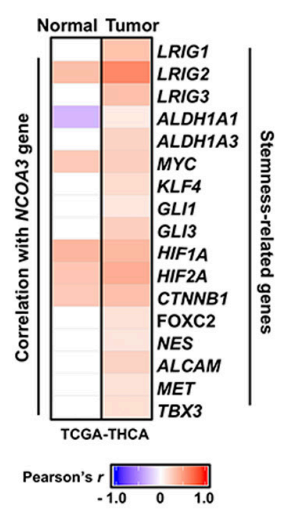

B-I

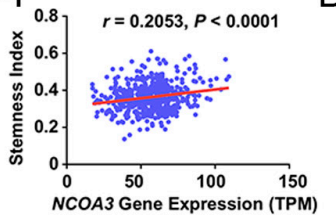

C-I

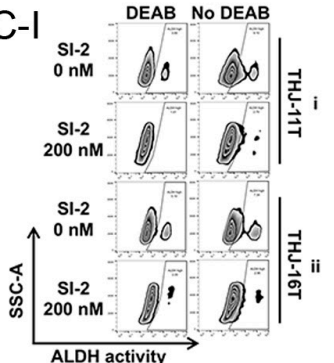

B-II

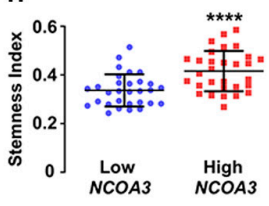

C-II

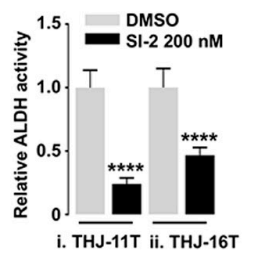

E-I

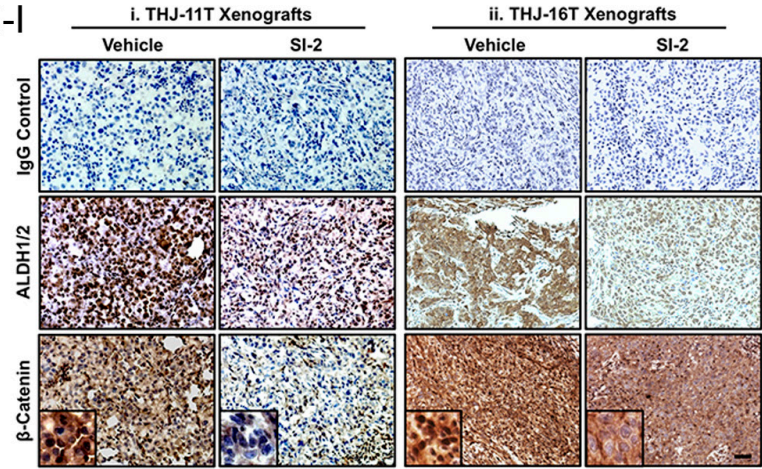

D-I
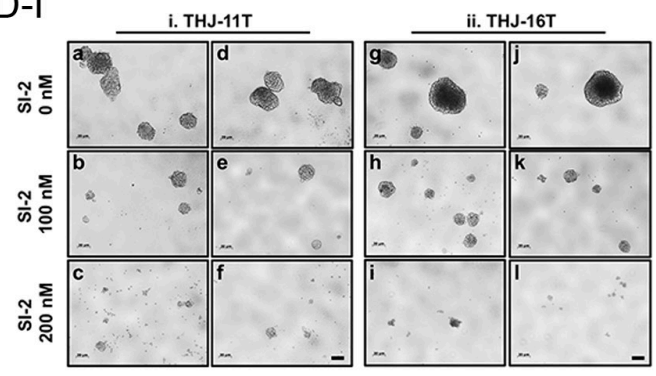

D-II

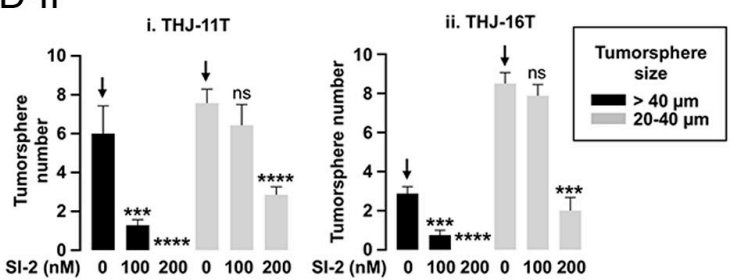

E-II

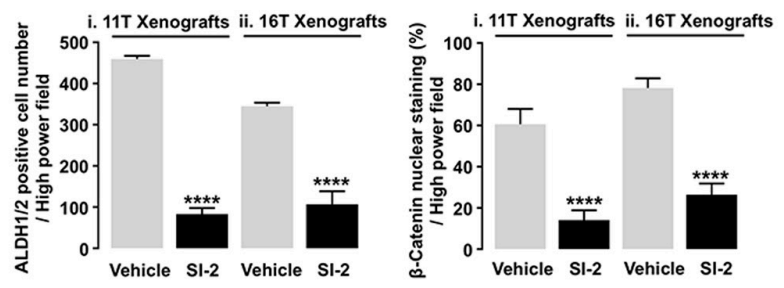

Figure 4

SI-2 can target cancer stem-like cells (CSCS) in ATC. (A) Strong positive relationship of NCOA3 with stemness-related genes in human thyroid cancer $(n=505)$, compared to normal thyroid tissue $(n=59)$; TCGA-THCA data analyses. (B) Positive correlation between NCOA3 and Stemness Index (I) and higher Stemness Index in tumor samples with high NCOA3 expression $(n=30)$ compared to tumors with low NCOA3 expression $(n=30)($ II); TCGA-THCA data analyses. (C) ALDEFLUOR assay (I) showing inhibitory effect of SI-2 on CSCS with high ALDH activity and its quantitative analysis (II, $n=4)$. (D) Tumor-sphere formation assay (I) showing inhibitory effect of SI-2 on the self-renewal ability of CSCs in ATC, and its quantitative analysis (II, $n=5$ ). (E) Representative IHC images and their magnified images ( $\times 3$ ) for CSCS markers (I) and quantitative analyses for the IHC results (II, $n=4)$ in the $11 \mathrm{~T}$ - and 16T-xenograft tumors treated with vehicle or SI-2. We used Pearson coefficient for the correlation analyses and two-tailed Student $t$-test for the comparison of Stemness Index between low and high NCOA3 tumor group. Significant differences were indicated by asterisks $(P<0.001$ ( $* \star *)$ and $P<0.0001(* * * *))$. Data represent the mean \pm S.D. Scale bar represents $20 \mu \mathrm{m}$. CSC, cancer stem-like cell; ATC, anaplastic thyroid cancer; IHC, immunohistochemistry; THCA, thyroid cancer; DEAB, diethylaminobenzaldehyde. A full color version of this figure is available at https://doi.org/10.1530/ ERC-19-0482.

THJ-16T cells, indicating the inhibitory effect of SI-2 on self-renewal ability of the both ATC cells (Fig. 4D-I-i and -ii, respectively). SI-2 treatment significantly decreased the number of CSC tumorsphere with size greater than $20 \mu \mathrm{m}$ but less than $40 \mu \mathrm{m}$ (63\% reduction in THJ-11T cells treated with SI-2 at concentrations of $200 \mathrm{nM}$; Fig. 4D-II-i, gray bars). Further, the number of tumorsphere with size greater than $40 \mu \mathrm{m}$ was reduced by $80 \%$ in cells treated with $100 \mathrm{nM}$ SI-2. No tumorspheres with a size greater than $40 \mu \mathrm{m}$ were detected with SI-2 at $200 \mathrm{nM}$ (Fig. 4D-II-i, black bars). Similar reduction patterns of tumorsphere formation were also detected in THJ-16T cells (Fig. 4D-I-ii). SI-2 treatment markedly decreased the number of CSC tumorsphere with a size greater than 40 $\mu \mathrm{m}$ by $75 \%$ in THJ-16T cells treated with $100 \mathrm{nM}$ of SI-2.
No tumorspheres with a size greater than $40 \mu \mathrm{m}$ were detected in cells treated with $200 \mathrm{nM}$ of SI-2 (Fig. 4D-II-ii, black bars). These results indicated that SI-2 effectively inhibited CSC capacity in ATC cells.

Using immunohistochemical staining, we also determined the protein abundance of ALDH1/2 and $\beta$-catenin in THJ-11T and THJ-16T tumors after treatment of SI-2 (Fig. 4E-I-i and -ii, respectively; middle panel for ALDH1/2; lower panel for $\beta$-catenin) as compared with controls. The number of cells positively stained with ALDH1/2 was significantly decreased by $82 \%$ and $69 \%$ in SI-2-treated THJ-11T and THJ-16T tumors versus vehicle-treated groups (Fig. 4E-II-i and -ii, left panel). There were significant $77 \%$ and $67 \%$ decreases in the $\beta$-catenin nuclear-stained cancer cells after SI-2 treatment in https://erc.bioscientifica.com

https://doi.org/10.1530/ERC-19-0482 (c) 2020 Society for Endocrinology Published by Bioscientifica Ltd. Printed in Great Britain 
THJ-11T and THJ-16T tumors, respectively (Fig. 4E-II-i and-ii, right panel). Altogether, these findings indicated that SRC-3 inhibition by SI-2 suppresses the CSC capacity in in vitro ATC culture cells and in mouse xenograft models.

\section{SRC-3 is highly expressed in human ATC tissues}

To assess whether SRC-3 is an appropriate target for treatment of patients with ATC, we compared the SRC-3 protein abundance among human normal thyroid tissue (Fig. 5A-I-a), FTC (5A-I-b), PTC (5A-I-c), and ATC (5A-I-d, -e, -f). The nuclear expression of SRC-3 was clearly higher in ATCs than in normal thyroid tissues (panel a vs panels b and c, Fig. 5A-II). Quantitative analysis shows that $54.6 \%$ of ATC cells were positive for SRC-3, whereas only $18.6 \%$ of PTC cells, $13.9 \%$ of FTC cells, and $18.3 \%$ of normal thyroid cells were positive for SRC-3 (Fig. 5A-III). To understand how elevated expression of SRC-3 could impact tumor cell proliferation, we next compared the nuclear expression of the proliferation marker, Ki-67, among human normal thyroid (Fig. 5B-I-a), PTC (5B-I-b), and ATC (5B-I-C). We found that more than $80 \%$ of ATC cells were positive for Ki-67, while only $7.3 \%$ of normal thyroid cells and $30.4 \%$ of PTC cells were positively stained for Ki-67 (Fig. 5B-II), suggesting that SRC-3 is critically involved in rapid and sustained proliferation of ATC cells.

We next validated the clinical significance of the NCOA3 gene in human thyroid cancer by analysis of the thyroid cancer database in TCGA. For this analysis, we first divided the data into two groups according to mRNA expression of NCOA3: low and high NCOA3 expression

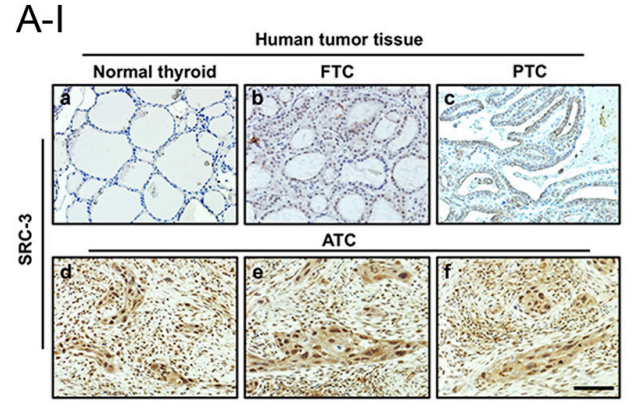

C

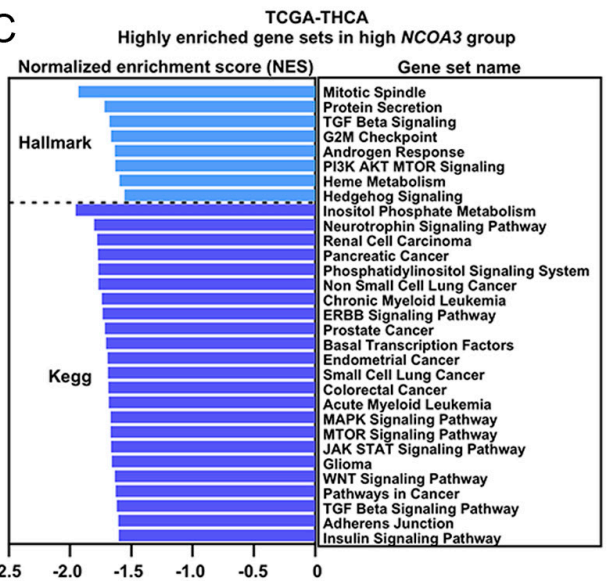

A-II

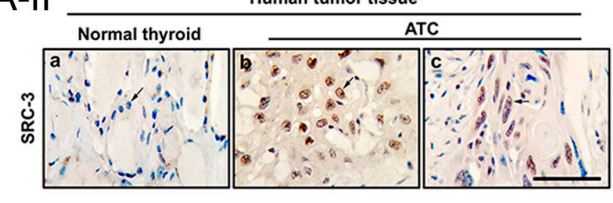

B-I

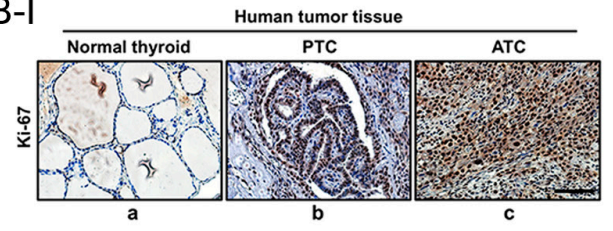

$\mathrm{D}$
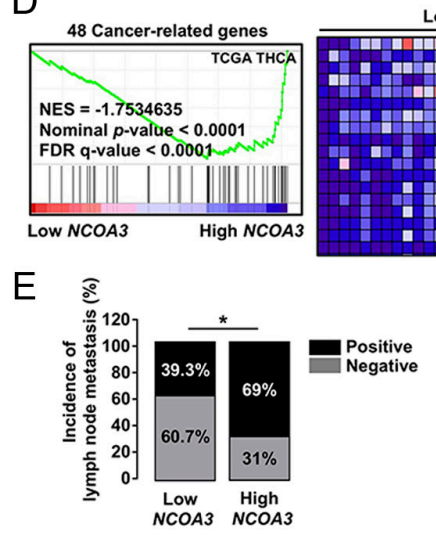

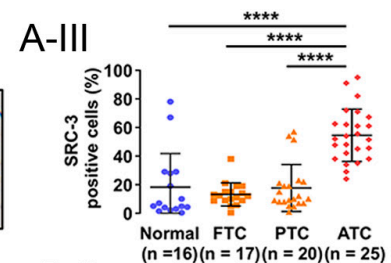

B-II

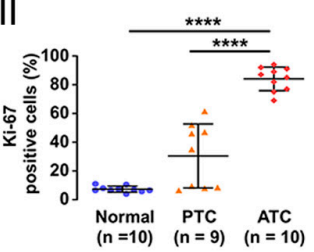

Low NCOA3

\section{Figure 5}

High expression of SRC-3 is closely related to thyroid cancer aggressiveness in human. (A) Representative IHC images for SRC-3 (I), their magnified images ( $\times 2$ ) showing nuclear staining of SRC-3 (II), and quantitative analysis for the IHC results (III) in different stages of human thyroid cancer. (B) Representative IHC images for Ki-67 (I) and quantitative analysis for the IHC results (II) in normal thyroid, PTC, and ATC. (C) Highly enriched Hallmark or Kegg gene sets related to multiple oncogenic pathways (nominal $P$-value $<0.05$ and FDR $q$-value $<0.25$ ) in high NCOA3 expression group, compared to low $N C O A 3$ group. (D) Coordinated enrichment of 48 cancer-related genes in high NCOA3 expression group. (E) Comparison of incidence of cervical lymph node metastasis between the low and the high NCOA3 groups by two-tailed $x^{2}$ test. (C, D, and E) TCGA-THCA data analyses. For the GSEA and clinical data analyses, we used the transcriptome data of tumors with top 30 and bottom 30 NCOA3 expression levels in the TCGA-THCA data (low vs high NCOA3 group; $n=30$, respectively). Significant differences were indicated by asterisks $\left(P<0.05\left(^{*}\right)\right.$ and $\left.P<0.0001(* * *)\right)$. Scale bar represents $50 \mu \mathrm{m}$. IHC, immunohistochemistry; FTC, follicular thyroid cancer; PTC, papillary thyroid cancer; ATC, anaplastic thyroid cancer; THCA. Thyroid cancer; GSEA, gene set enrichment analysis. A full color version of this figure is available at https://doi.org/10.1530/ERC-19-0482. 
group ( $n=30$, respectively), and then performed GSEA to identify biological characteristics of the high NCOA3 group, compared to the low $N C O A 3$ group. Interestingly, we found that multiple oncogenic pathways including PI3K-AKT-MTOR and MAPK signaling involved in various types of human cancer were enriched in the high NCOA3 expression group (Fig. 5C; see also Supplementary Tables 1 and 2, see section on supplementary materials given at the end of this article), while there was no significantly enriched gene set in the low NCOA3 group (Supplementary Table 3). This result indicates extensive involvement of SRC-3 in the activation of multiple oncogenic pathways for cancer progression. To confirm this finding, we next created our own gene set including 48 cancer driver- and pathway-related genes, as previously reported (Mlecnik et al. 2016) and performed GSEA using this gene set. Consistently, high $\mathrm{NCOA} 3$ group exhibited coordinated enrichment of these 48 cancer-related genes (Fig. 5D). Of note, incidence of cervical lymph node metastasis, an important prognostic factor in thyroid cancer, was more frequent in the high than the low NCOA3 expression group (69.0\% vs 39.3\%; Fig. 5E). Together, these data indicated that SRC-3 would be a potential therapeutic target in human ATC against which SI-2 could be tested in future clinical trials.

\section{Discussion}

SRC-3 activates transcription of nuclear receptors and other transcriptional factors by assembling into a multiprotein coactivator complex on the target genes (Yan et al. 2006, Wang et al. 2016). Aberrant expression of SRC-3 was found in many human cancers, and the oncogenic roles of SRC-3 were demonstrated in cell-based studies and in vivo mouse models. That SRC-3 deficiency in a mouse model of thyroid cancer (Thr $b^{P V / P V}$ mice) led to increased survival and delayed metastatic spread shows that SRC-3 could be a potential therapeutic target in thyroid cancer. Thus, in the present study, we targeted SRC-3 in ATC by using a newly developed selective SRC-3 small molecule inhibitor, SI-2. The therapeutic effects of SI-2 were demonstrated in breast cancer cells. SI-2 selectively killed breast cancer cells at the low nanomolar range of IC-50 values without adverse effects on the survival of normal cells (Song et al. 2016). Our present study shows that SRC-3 expression was significantly increased in human ATC tissues and that SRC-3 inhibition by SI-2 decreased cancer cell survival and tumor growth through induction of apoptosis and impeding cell cycle progression in in vitro and in vivo studies. These present preclinical findings indicate that targeting SRC-3 by SI-2 may be a potentially effective strategy for treating patients with ATC.

Emerging evidence suggests that cancer stem-like cells (CSCs) may initiate new tumors in ATC. CSCs are a subpopulation of cells in tumors that share molecular similarities to embryonic or adult stem cells and are frequently related to chemo- and radio-resistance (Reya et al. 2001, Lin 2011). A previous study reported that $9.4 \%$ of THJ-11T cells and 4.5\% of THJ-16T cells are enriched CSCs (Li et al. 2013). CSCs in thyroid cancers were also shown to express high levels of ALDH (Todaro et al. 2010). Consistent with these reports, our study also showed that a subpopulation of THJ-11T and THJ-16 cells possessed the ability to form tumor spheres and exhibited high expression of ALDH, characteristics of CSCs. Analysis of the TCGA thyroid cancer database clearly indicated a close association of NCOA3 (SRC-3) gene expression with stemness-related genes (Fig. 4A). These findings prompted us to evaluate whether SI-2 could be effective to suppress CSCs via targeting SRC-3. Remarkably, we found that SI-2 was effective in reducing not only the number of tumor spheres, but also their size. Moreover, SI-2 also inhibited ALDH expression and activity in ATC cells. The decreases in tumor sphere formation and reduced expression of ALDH are indicative of reduced CSC activity.

That SI-2 suppressed CSCs in ATC, as demonstrated in the present study, has important clinical implications. ATC is one of the most aggressive solid tumors in humans. Currently there is no effective treatment. One possible explanation of resistance to treatment in ATC could be the perpetual self-renewing and re-initiating development of tumors driven by CSCs. The finding that SI-2 could suppress the CSC capacity suggested that SI- 2 treatment is useful for both monotherapy or in combination therapy to overcome potential resistance due to CSC cells.

It is important to point out that THJ-11T cells and THJ-16 cells have distinct genetic lesions. THJ-11T cells harbor a KRASG12V mutation, whereas THJ-16 cells express PI3KE454K, TP53, and Rb mutations (Marlow et al. 2010). In spite of these distinct driver mutations, SI-2 had similar efficacy in the inhibition of tumor cell growth in both ATC cells, as shown in in vitro and in vivo analyses. These observations suggest that aberrant signaling initiated by different upstream driver mutations would converge in the transcription responses. Other components involved in the transcription machinery such as chromatin structure, transcription mediator, and polymerase complexes would be critical in the final manifestation of oncogenic responses. SRC-3, a critical 
transcription activator, would be an effective target to suppress these aberrant transcriptional responses. Recent global genomic analyses have demonstrated that in ATC, in addition to mutations in TERT promoter, TP53, PI3K/AKT/mTOR pathway effectors, mutations in SWI/ SNF subunits, histone methyltransferases, and EIF1AX (a component of the translational preinitiation complex) were also detected (Landa et al. 2016). The identification of small molecular inhibitors such as SI-2 to target SRC-3 or other transcription regulators could be promising approaches for effective ATC treatment and prevention.

\section{Supplementary materials}

This is linked to the online version of the paper at https://doi.org/10.1530/ ERC-19-0482.

\section{Declaration of interest}

Dr O'Malley and Lonard have equity and research contract funding from Coactigon, Inc. The other authors have nothing to disclose.

\section{Funding}

This research was supported by the Intramural Research Program of the Center for Cancer Research, National Cancer Institute, National Institutes of Health and BWO (HD08818, DK113954).

\section{Author contribution statement}

Conception and design was performed by W-K Lee, W-G Kim, B W O'Malley, and S-y Cheng. Development of methodology was performed by W-K Lee, W-G Kim, L Fozzatti, S Park, and L Zhao. Acquisition of data (provided animals, acquired and managed patients, provided facilities, etc.) was performed by W-K Lee, W-G Kim, S Park, and L Zhao. Analysis and interpretation of data (e.g. statistical analysis, biostatistics, and computational analysis) was performed by W-K Lee, W-G Kim, S Park, M C Willingham, and S-y Cheng. Writing, review, and/or revision of the manuscript was performed by $\mathrm{W}-\mathrm{K}$ Lee, W-G Kim, S-y Cheng. Administrative, technical, or material support (i.e. reporting or organizing data and constructing databases) was performed by L Zhao, M C Willingham, B W O'Malley, D Lonard, and S-y Cheng. Study supervision was performed by S-y Cheng.

\section{References}

Al-Hajj M, Wicha MS, Benito-Hernandez A, Morrison SJ \& Clarke MF 2003 Prospective identification of tumorigenic breast cancer cells. PNAS 100 3983-3988. (https://doi.org/10.1073/pnas.0530291100)

Anzick SL, Kononen J, Walker RL, Azorsa DO, Tanner MM, Guan XY, Sauter G, Kallioniemi OP, Trent JM \& Meltzer PS 1997 AIB1, a steroid receptor coactivator amplified in breast and ovarian cancer. Science 277 965-968. (https://doi.org/10.1126/science.277.5328.965)

Furumoto H, Ying H, Chandramouli GV, Zhao L, Walker RL, Meltzer PS, Willingham MC \& Cheng SY 2005 An unliganded thyroid hormone beta receptor activates the cyclin D1/cyclin-dependent kinase/ retinoblastoma/E2F pathway and induces pituitary tumorigenesis.
Molecular and Cellular Biology 25 124-135. (https://doi.org/10.1128/ MCB.25.1.124-135.2005)

Guigon CJ, Zhao L, Willingham MC \& Cheng SY 2009 PTEN deficiency accelerates tumour progression in a mouse model of thyroid cancer. Oncogene 28 509-517. (https://doi.org/10.1038/onc.2008.407)

Haddad RI, Lydiatt WM, Ball DW, Busaidy NL, Byrd D, Callender G, Dickson P, Duh QY, Ehya H, Haymart M, et al. 2015 Anaplastic thyroid carcinoma, version 2.2015. Journal of the National Comprehensive Cancer Network 13 1140-1150. (https://doi. org/10.6004/jnccn.2015.0139)

Hoelting T, Zielke A, Siperstein AE, Clark OH \& Duh QY 1994 Aberrations of growth factor control in metastatic follicular thyroid cancerin vitro. Clinical and Experimental Metastasis 12 315-323. (https://doi.org/10.1007/bf01753838)

Jeon MJ, Chun SM, Kim D, Kwon H, Jang EK, Kim TY, Kim WB, Shong YK, Jang SJ, Song DE, et al. 2016 Genomic alterations of anaplastic thyroid carcinoma detected by targeted massive parallel sequencing in a BRAF(V600E) mutation-prevalent area. Thyroid 26 683-690. (https://doi.org/10.1089/thy.2015.0506)

Kim CS, Vasko VV, Kato Y, Kruhlak M, Saji M, Cheng SY \& Ringel MD 2005 AKT activation promotes metastasis in a mouse model of follicular thyroid carcinoma. Endocrinology 146 4456-4463. (https:// doi.org/10.1210/en.2005-0172)

Kim WG, Guigon CJ, Fozzatti L, Park JW, Lu C, Willingham MC \& Cheng SY 2012 SKI-606, an Src inhibitor, reduces tumor growth, invasion, and distant metastasis in a mouse model of thyroid cancer. Clinical Cancer Research 18 1281-1290. (https://doi. org/10.1158/1078-0432.CCR-11-2892)

Kim WG, Zhu X, Kim DW, Zhang L, Kebebew E \& Cheng SY 2013 Reactivation of the silenced thyroid hormone receptor beta gene expression delays thyroid tumor progression. Endocrinology 154 25-35. (https://doi.org/10.1210/en.2012-1728)

Kim WG, Zhao L, Kim DW, Willingham MC \& Cheng SY 2014 Inhibition of tumorigenesis by the thyroid hormone receptor beta in xenograft models. Thyroid 24 260-269. (https://doi.org/10.1089/ thy.2013.0054)

Landa I, Ibrahimpasic T, Boucai L, Sinha R, Knauf JA, Shah RH, Dogan S, Ricarte-Filho JC, Krishnamoorthy GP, Xu B, et al. 2016 Genomic and transcriptomic hallmarks of poorly differentiated and anaplastic thyroid cancers. Journal of Clinical Investigation 126 1052-1066. (https://doi.org/10.1172/JCI85271)

Li W, Reeb AN, Sewell WA, Elhomsy G \& Lin RY 2013 Phenotypic characterization of metastatic anaplastic thyroid cancer stem cells. PLOS ONE 8 e65095. (https://doi.org/10.1371/journal.pone.0065095)

Lin RY 2011 Thyroid cancer stem cells. Nature Reviews: Endocrinology 7 609-616. (https://doi.org/10.1038/nrendo.2011.127)

Lonard DM \& O'Malley BW 2006 The expanding cosmos of nuclear receptor coactivators. Cell 125 411-414. (https://doi.org/10.1016/j. cell.2006.04.021)

Lonard DM \& O'Malley BW 2012 Nuclear receptor coregulators: modulators of pathology and therapeutic targets. Nature Reviews: Endocrinology 8 598-604. (https://doi.org/10.1038/nrendo.2012.100)

Louie MC, Zou JX, Rabinovich A \& Chen HW 2004 ACTR/AIB1 functions as an E2F1 coactivator to promote breast cancer cell proliferation and antiestrogen resistance. Molecular and Cellular Biology 24 5157-5171. (https://doi.org/10.1128/MCB.24.12.51575171.2004)

Malta TM, Sokolov A, Gentles AJ, Burzykowski T, Poisson L, Weinstein JN, Kamińska B, Huelsken J, Omberg L, Gevaert O, et al. 2018 Machine learning identifies stemness features associated with oncogenic dedifferentiation. Cell 173 338.e15-354.e15. (https://doi. org/10.1016/j.cell.2018.03.034)

Marlow LA, D'Innocenzi J, Zhang Y, Rohl SD, Cooper SJ, Sebo T, Grant C, McIver B, Kasperbauer JL, Wadsworth JT, et al. 2010 Detailed molecular fingerprinting of four new anaplastic thyroid carcinoma cell lines and their use for verification of RhoB as a 
molecular therapeutic target. Journal of Clinical Endocrinology and Metabolism 95 5338-5347. (https://doi.org/10.1210/jc.2010-1421)

Mlecnik B, Bindea G, Kirilovsky A, Angell HK, Obenauf AC, Tosolini M, Church SE, Maby P, Vasaturo A, Angelova M, et al. 2016 The tumor microenvironment and Immunoscore are critical determinants of dissemination to distant metastasis. Science Translational Medicine 8 327ra26. (https://doi.org/10.1126/scitranslmed.aad6352)

Molinaro E, Romei C, Biagini A, Sabini E, Agate L, Mazzeo S, Materazzi G, Sellari-Franceschini S, Ribechini A, Torregrossa L, et al. 2017 Anaplastic thyroid carcinoma: from clinicopathology to genetics and advanced therapies. Nature Reviews: Endocrinology 13 644-660. (https://doi.org/10.1038/nrendo.2017.76)

Mussi P, Yu C, O'Malley BW \& Xu J 2006 Stimulation of steroid receptor coactivator-3 (SRC-3) gene overexpression by a positive regulatory loop of E2F1 and SRC-3. Molecular Endocrinology 20 3105-3119. (https://doi.org/10.1210/me.2005-0522)

Nevins JR, Leone G, DeGregori J \& Jakoi L 1997 Role of the Rb/E2F pathway in cell growth control. Journal of Cellular Physiology 173 233-236. (https://doi.org/10.1002/(SICI)10974652(199711)173:2<233::AID-JCP27>3.0.CO;2-F)

Reya T, Morrison SJ, Clarke MF \& Weissman IL 2001 Stem cells, cancer, and cancer stem cells. Nature $\mathbf{4 1 4} 105-111$. (https://doi. org/10.1038/35102167)

Rohira AD, Yan F, Wang L, Wang J, Zhou S, Lu A, Yu Y, Xu J, Lonard DM \& O'Malley BW 2017 Targeting SRC coactivators blocks the tumor-initiating capacity of cancer stem-like cells. Cancer Research 77 4293-4304. (https://doi.org/10.1158/0008-5472.CAN-162982)

Smallridge RC 2012 Approach to the patient with anaplastic thyroid carcinoma. Journal of Clinical Endocrinology and Metabolism 97 2566-2572. (https://doi.org/10.1210/jc.2012-1314)

Smallridge RC, Ain KB, Asa SL, Bible KC, Brierley JD, Burman KD, Kebebew E, Lee NY, Nikiforov YE, Rosenthal MS, et al. 2012 American Thyroid Association guidelines for management of patients with anaplastic thyroid cancer. Thyroid 22 1104-1139. (https://doi.org/10.1089/thy.2012.0302)

Song X, Chen J, Zhao M, Zhang C, Yu Y, Lonard DM, Chow DC, Palzkill T, Xu J, O'Malley BW, et al. 2016 Development of potent small-molecule inhibitors to drug the undruggable steroid receptor coactivator-3. PNAS 113 4970-4975. (https://doi.org/10.1073/ pnas.1604274113)

Subbiah V, Kreitman RJ, Wainberg ZA, Cho JY, Schellens JHM, Soria JC, Wen PY, Zielinski C, Cabanillas ME, Urbanowitz G, et al. 2018 Dabrafenib and trametinib treatment in patients with locally advanced or metastatic BRAF V600-mutant anaplastic thyroid cancer. Journal of Clinical Oncology 36 7-13. (https://doi.org/10.1200/ JCO.2017.73.6785)

Suzuki H, Willingham MC \& Cheng SY 2002 Mice with a mutation in the thyroid hormone receptor $\beta$ gene spontaneously develop thyroid carcinoma: a mouse model of thyroid carcinogenesis. Thyroid $\mathbf{1 2}$ 963-969. (https://doi.org/10.1089/105072502320908295)

Tiedje V, Stuschke M, Weber F, Dralle H, Moss L \& Fuhrer D 2018 Anaplastic thyroid carcinoma: review of treatment protocols. Endocrine-Related Cancer 25 R153-R161. (https://doi.org/10.1530/ ERC-17-0435)

Todaro M, Iovino F, Eterno V, Cammareri P, Gambara G, Espina V, Gulotta G, Dieli F, Giordano S, De Maria R, et al. 2010 Tumorigenic and metastatic activity of human thyroid cancer stem cells. Cancer Research 70 8874-8885. (https://doi.org/10.1158/0008-5472. CAN-10-1994)

Torres-Arzayus MI, Font de Mora J, Yuan J, Vazquez F, Bronson R, Rue M, Sellers WR \& Brown M 2004 High tumor incidence and activation of the PI3K/AKT pathway in transgenic mice define AIB1 as an oncogene. Cancer Cell 6 263-274. (https://doi.org/10.1016/j. ccr.2004.06.027)

Trimarchi JM \& Lees JA 2002 Sibling rivalry in the E2F family. Nature Reviews: Molecular Cell Biology 3 11-20. (https://doi.org/10.1038/ nrm714)

Wang L, Lonard DM \& O'Malley BW 2016 The role of steroid receptor coactivators in hormone dependent cancers and their potential as therapeutic targets. Hormones and Cancer 7 229-235. (https://doi. org/10.1007/s12672-016-0261-6)

Wells JA \& McClendon CL 2007 Reaching for high-hanging fruit in drug discovery at protein-protein interfaces. Nature $\mathbf{4 5 0} 1001-1009$. (https://doi.org/10.1038/nature06526)

Xu J, Wu RC \& O'Malley BW 2009 Normal and cancer-related functions of the p160 steroid receptor co-activator (SRC) family. Nature Reviews: Cancer 9 615-630. (https://doi.org/10.1038/nrc2695)

Yan J, Tsai SY \& Tsai MJ 2006 SRC-3/AIB1: transcriptional coactivator in oncogenesis. Acta Pharmacologica Sinica 27 387-394. (https://doi. org/10.1111/j.1745-7254.2006.00315.x)

Ying H, Willingham MC \& Cheng SY 2008 The steroid receptor coactivator-3 is a tumor promoter in a mouse model of thyroid cancer. Oncogene 27 823-830. (https://doi.org/10.1038/sj. onc.1210680)

Zhu X, Park S, Lee WK \& Cheng SY 2019 Potentiated anti-tumor effects of BETi by MEKi in anaplastic thyroid cancer. Endocrine-Related Cancer 27 739-750. (https://doi.org/10.1530/ERC-19-0107)

Received in final form 22 January 2020

Accepted 24 January 2020

Accepted Manuscript published online 24 January 2020 (c) 2020 Society for Endocrinology Published by Bioscientifica Ltd. Printed in Great Britain 